\title{
Simultaneous release of two drugs from polymer nano-implant inhibits recurrence in glioblastoma spheroids
}

Greeshma Devassy ${ }^{1}$, Ranjith Ramachandran ${ }^{1}$, Kottarapat Jeena ${ }^{1}$, Vijayabhaskar R. Junnuthula ${ }^{1}$, Vindya K. Gopinatha ${ }^{2}$, Cheripelil A. Manju ${ }^{1}$, Maneesh Manohar ${ }^{1}$, Shantikumar V. Nair ${ }^{1}$, Sathees C Raghavan $^{2 *}$ and Manzoor Koyakutty ${ }^{1 *}$

*These authors contributed equally to this work.

${ }^{1}$ Amrita Centre for Nanosciences and Molecular Medicine, Amrita Vishwa Vidyapeetham, Amrita Institute of Medical Sciences, Kochi 682 041, India.

${ }^{2}$ Department of Biochemistry, Indian Institute of Science Bangalore, 560012, India.

Submitted: November 27, 2018

Accepted: December 21, 2018

Published: December 22, 2018

\section{Graphical Abstract}

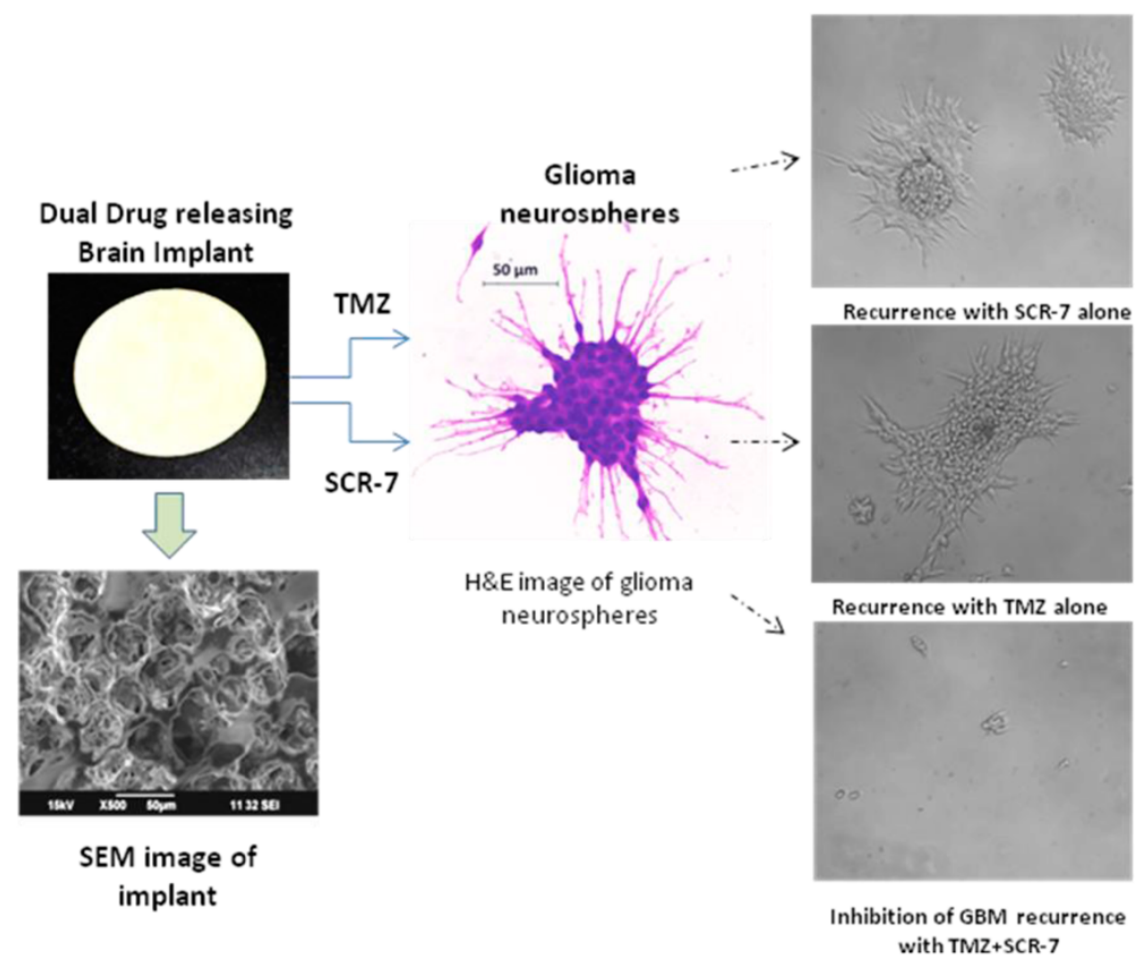

\section{Abstract}

Local implant-based delivery of rationally selected combination of chemotherapeutics has some major advantages for the treatment of glioblastoma such as: (a) $100 \%$ bio-availability locally in brain can be achieved at the tumor site (b) avoid systemic leakage and associated toxicity, and (c) simultaneous inhibition of multiple, mutually exclusive cancer mechanisms is possible. Here, we report a polymeric brain implant capable of delivering two different drugs in recurrent glioma cells. We have selected a combination of clinically used DNA alkylating agent, Temozolomide, and a DNA mismatch repair protein (Ligase IV) inhibitor, SCR-7, and delivered simultaneously into tumor spheroids formed by rat

* Corresponding Authors: Dr. Manzoor Koyakutty, Ph.D, Amrita Centre for Nanosciences and Molecular Medicine, Amrita Vishwa Vidyapeetham, Amrita Institute of Medical Sciences, Kochi 682 041, India. Email: manzoork@aims.amrita.edu; Dr. Sathees C Raghavan, Ph.D., Department of Biochemistry, Indian Institute of Science Bangalore, 560012, India. E-mail: sathees@biochem.iisc.ernet.in 
glioma cells, C6. The dual-drug loaded polymeric wafer, prepared by lyophilization method, could deliver both the drugs in a controlled fashion. To test the efficacy of this system, we have optimized an in vitro recurrent model of glioma spheroids wherein, the implant released both the drugs in a sustained fashion, thereby continuously exposing the cells to DNA methylation while inhibiting the DNA repair pathways. This leads to synergistic toxicity and inhibition of tumor recurrence for extended duration compared to free drug combination.

Keywords:

SCR7, temozolomide, neurospheres, Polymer implants, glioblastoma

\section{Abbreviations}

- $\quad$ SCR7

- TMZ: temozolomide

\section{Rationale and Purpose}

Local recurrence is one of the major challenges in high grade glioma. Prolonged delivery of multiple drugs using nano polymer implants that can enhance the anti-tumoral activity locally in brain will have significant impact in anti-glioma therapy.

\section{Introduction}

Glioma is one of the most devastating primary brain tumors with median survival of 12-15 months. ${ }^{1}$ Even with recent advancements in surgery, radiation therapy, and chemotherapy, the five-year survival is less than $5 \%{ }^{2}$ Current clinical drugs are temozolomide (TMZ), given orally, and BCNU given as intracranial implant (Gliadel $^{\mathrm{TM}}$ ). Systemic TMZ therapy faces the challenge of penetrating the blood brain barrier (BBB) which can lead to the lack of an effective concentration of the drug reaching the target brain tissue. Drawbacks of the Gliadel ${ }^{\mathrm{TM}}$ wafer are a short-sustained release of one week and limited tissue penetration. Tumor recurrence is a prime factor deciding patient prognosis, which is almost inevitable for glioma and has been attributed to drug resistances arising from genomic mutations such as EGFR vIII, IDH, MGMT promoter methylation, etc. ${ }^{3-8}$ In addition, cancer stem cells are another important factor responsible for recurrence due to its capability to repopulate glioma cells even after treatment and also contributes to drug resistance from altered DNA repair mechanisms. Multiple molecular mechanisms play a role in maintaining cancer stem cells, which makes it difficult to target with a single chemotherapeutic agent. ${ }^{9-14}$ It is also reported that in almost $90 \%$ of glioma patients recurrence is at the original tumor location within a depth of 2 to $3 \mathrm{~cm}$ from the resection margin. ${ }^{15}$
To address these multifactorial challenges, multiple chemotherapeutic molecules may need to be delivered together; for example, one for tumor kill, and another to address resistance mechanisms. However, attaining therapeutic concentration of various drugs simultaneously at the tumor vicinity, by crossing BBB, is a major challenge when given systemically. One interesting option for delivering a suitable combination of drugs targeted to different cancer mechanisms is the use of biodegradable, drugloaded brain implants that can direct 100\% delivery in the tumor resected cavity in a controlled fashion. ${ }^{16-19}$ Polymer drug delivery systems which include drug eluting rods, gels, wafers, nanoparticles, and microcapsules have drawn significant attention over the last few decades. ${ }^{20-23}$ However, Gliadel ${ }^{\mathrm{TM}}$ is the only FDA-approved intracranial implant available in clinic which delivers cytotoxic drug BCNU [1,3-bis (2-chloroethyl)-1-nitrosourea]. Although median survival was improved by $\sim 2.3$ months as a result of 7 days of BCNU release from Gliadel ${ }^{\mathrm{TM}}$, tumor recurrence was reported in almost all patients. The short half-life of $\mathrm{BCNU}$, its limited tissue penetration capacity, and short duration of release (7 days) are proposed as possible reasons for the limited success of Gliadel ${ }^{\mathrm{TM}} .^{24-26}$ To combat these challenges we recently developed an electrospun polymeric wafer eluting TMZ from 7 days up to one month in brain resulting in prolonged survival for $85 \%$ of treated tumor-bearing animals. ${ }^{27}$ However, recurrence was seen in $15 \%$ of animals indicating that the TMZ-resistant population needs to be addressed judiciously by combining with other novel molecules which can target the DNA repair pathways in the TMZ-resistant population. 
In this study, we developed a dual-drug releasing polymer implant loaded with TMZ together with a novel DNA mismatch repair protein (Ligase IV) inhibitor; SCR-7. ${ }^{28-31} \mathrm{TMZ}$ induces DNA damage by adding methyl groups on the $\mathrm{O} 6$ position of guanine residues. This will cause futile cycles of DNA repair (mismatch repair) leading to the accumulation of double strand breaks (DSB) triggering apoptosis-mediated cell death. ${ }^{28-30}$ SCR-7 is an inhibitor of non-homologous end joining (NHEJ), one of the primary mechanisms of DSB repair. $^{31-33}$ It blocks NHEJ by inhibiting Ligase IV, a mismatch repair protein, leading to the accumulation of DSBs and activates apoptotic pathways. ${ }^{34-35}$ We hypothesize that SCR7 will enhance the cytotoxicity caused by TMZ, by arresting the ligase IV mediated DSB repair, when delivered together. Thus, by inhibiting the DNA repair mechanism, we propose to improve the sensitivity of cells towards alkylating agents. To deliver TMZ and SCR 7 together, we developed a polymeric brain implant using simple lyophilization method where both the drugs are co-loaded in appropriate concentrations. Moreover, we have developed an in vitro tumor recurrent assay using glioma spheroids wherein the cells survived after the treatment with the test samples were further cultured for 3-12 days to check its ability to recur and form tumor spheroids.

\section{Materials and methods}

All the chemicals and reagents used in this study were obtained from Sigma (St. Louis, U.S.A.) unless specified otherwise. Temozolomide was purchased from AK Scientific, USA, SCR-7 was synthesized as described before, ${ }^{31}$ PLGA (75:25) molecular weight 17,000-20,000 was purchased from Wako Pure Chemical Industries, Ltd., Japan. PLA molecular weight 1,50,000 was purchased from Goodfellow Cambridge Ltd (England).

\section{Experiments}

\section{In vitro cytotoxicity assessment.}

C6 rat glioma cell line and T98G MGMT over-expressing human glioma cell line were procured from National Centre for Cell Science, Pune, India. The cells were maintained in Dulbecco's Modified Eagle's Medium (DMEM; Invitrogen, CA, USA) supplemented with 10\% Fetal Bovine Serum (FBS; Invitrogen, CA, USA) and with $50 \mathrm{IU} / \mathrm{mL}$ penicillin and 50 $\mu \mathrm{g} / \mathrm{ml}$ streptomycin. Cells were incubated in a humidified atmosphere of $5 \% \mathrm{CO}_{2}$ at $37^{\circ} \mathrm{C}$. Cell proliferation was measured using MTT colorimetric assay. For cytotoxicity assessment of free drug combinations, $\mathrm{C} 6$ cells were seeded in 96 well plates at a seeding density of $1 \times 10^{3}$ cells per well. All concentrations were used in triplicates. After a treatment period of 72 hours, supernatant was removed and cells were washed with PBS. 10\% Next, MTT reagent in medium was added and plates were further incubated for 4 hours at $37^{\circ} \mathrm{C}$ in humidified incubator. After incubation solubilizing buffer is added to dissolve the formazan crystal and mixed well. Absorbance was read at 570 and $660 \mathrm{~nm}$ using microplate reader (PowerWave XS, BioTek, Vermont, U.S.A.). In vitro cytotoxicity assessment of TMZ and SCR7 polymer wafer combination were carried out in comparison with two sets of control groups (i) wafer group (namely bare wafer, TMZ wafer, SCR7 wafer and TMZ+SCR7 wafer) and (ii) free drug group (namely media alone, TMZ, SCR7 and TMZ+SCR7). C6 cells were seeded in 24 well plate at a seeding density of $5 \times 10^{3}$ cells per well. MTT assay was performed as mentioned above.

\section{Colony / neurosphere formation assay.}

C6 rat glioma cells were grown in serum free DMEM supplemented with EGF $(20 \mathrm{ng} / \mathrm{mL}$, R\&D Systems Germany), FGF (20 ng/mL, R\&D Systems Germany) and B27 $(20 \mu \mathrm{g} / \mathrm{mL}$, Gibco USA). Cells were seeded at seeding density of $5 \times 10^{3}$ cells per well in 24 well plate. At 24 hours following the seeding, cells were treated with drug/wafer containing media and maintained at $37^{\circ} \mathrm{C}$ in humidified incubator for 12 days without media change. In the wafer group, wafers were weighted and added so that the final doses would be $550 \mu \mathrm{M} \mathrm{TMZ}$ and $100 \mu \mathrm{M}$ SCR7. Photographs were taken at regular intervals using Nikon Eclipse TE2000U at $10 \times$ magnification. MTT standard curve was plotted for quantitative estimation of cells in the neurosphere. $\mathrm{C} 6$ cells were seeded from a range of $0.1 \times 10^{3}$ to $1.4 \times 10^{4}$. MTT reagent was added and incubated for 4 hours at $37^{\circ} \mathrm{C}$ in a humidifier. Formazan crystal solubilization was carried out and absorbance was read at specific wavelengths and a standard curve was plotted. For plotting cell growth curve, neurospheres from each time point were treated with MTT 
and a cell number was obtained from corresponding absorbance value in the standard curve.

\section{Apoptosis assay}

To determine apoptosis, treated C6 cells were stained with Annexin V and propidium iodide (PI) using Alexa Fluor Annexin V/Dead Cell Apoptosis Kit (Thermofisher, USA) 72 hours following incubation. Camptothecin was used as positive control and untreated cells as negative control. Assay performed according to manufacturer's instruction. Stained cells were analyzed by flow cytometry (FACS Aria, BD Biosciences, US)

\section{Characterization of spheres}

For studying cell viability within the spheroids, live dead assay was performed using live/dead cell viability assay kit (Thermofisher, USA) following manufacturer's instruction and viewed under fluorescent microscope (Leica DMI 3000). H \& E staining was done for neurospheres. C6 spheroids were grown on acidetched coverslips. Spheroids were then formalin fixed, dehydrated, and stained with modifications in the conventional H\&E staining protocol. For immunostaining for nestin, spheres were grown on cover slips, fixed with 4\% PFA (paraformaldehyde), permeabilized using 1\% triton, and blocked using 1\% FBS. Cover slips were then incubated with specific antibody overnight at $4^{\circ} \mathrm{C}$, washed and counter stained with PI. Spheres were imaged using confocal microscope.

\section{Preparation of TMZ and SCR7 loaded wafer.}

PLGA:PLA wafer was synthesized by lyophilization method. PLGA: PLA in ratio 25:75 were dissolved in Dichloro Methane (DCM) by stirring at 600 RPM for approximately 5 hours. Once dissolved, ethanol was added to the polymer solution and mixed well by stirring for 15 minutes. The blended polymer solution is then poured in to a glass plate, snap frozen using liquid nitrogen and kept for lyophilization (LABCONCO, Care Biosystems India Ltd). Polymer film thus obtained was stored at $4^{\circ} \mathrm{C}$. For the preparation of TMZ-loaded wafers, $10 \% \mathrm{TMZ}$ was dissolved in DCM along with PLGA: PLA. The wafer was prepared in the way mentioned for bare wafers. Similarly for SCR7 loaded wa- fer, 5\% SCR7 was dissolved in DCM and wafers synthesized in the same method as mentioned above.

\section{Characterization of lyophilized wafer}

Scanning electron microscope analysis was carried out to study the micro morphology, porosity and surface texture of the wafer. A piece of wafer was mounted on a metallic stub using double sided adhesive tape, coated with gold using Auto Fine coater (JFC-1600 JEOL, Japan) and finally imaged by SEM (JSM-6490LA JEOL, Japan). The elemental composition of the wafer was studied by Energy Dispersive Spectral Analysis using JEOL JSM-6490LA system and the spectral images were overlaid with the SEM image at $3000 \times$ magnification. The analysis was performed at a counting rate of $1898 \mathrm{cps}$ under accelerating voltage of $15 \mathrm{kV}$ for a sweep count rate of 26 after mounting samples on aluminum stub. FTIR spectra over the wavelength range $4000-400 \mathrm{~cm}^{-1}$ was recorded for drug-loaded wafers and also free drug using FTIR spectrometer IRAffinity 1S (Shimadzu, Scientific Instruments, Princeton, NJ, USA)

\section{Estimation of drug loading}

HPLC was performed to determine the amount of drug loaded in the wafer. A known amount of drug-loaded wafer was dissolved in $0.5 \mathrm{~mL}$ of chloroform. After dissolving completely, an equal volume of methanol was added and mixed well. To this methanol chloroform mix, $0.5 \mathrm{~mL}$ of deionized water was added, and centrifuged at $6000 \mathrm{rpm}$ for 10 minutes to get a phase separation. The methanol-water upper phase containing the drug was carefully separated and HPLC analysis was performed. The HPLC system consisted of a Shimadzu LC-9A pump and a Model 3200 PDA detector (Shimadzu, Scientific Instruments, Princeton, NJ, USA) set at $330 \mathrm{~nm}$. Separation was accomplished on a Qualisil GOLD, ODS, $5 \mu \mathrm{m}$, $150 \times 4.6 \mathrm{~mm} \mathrm{C18}$ column. For TMZ, mobile phase consisted of $0.5 \%$ glacial acetic acidmethanol (90:10, v:v) and was delivered at 1.0 $\mathrm{mL} / \mathrm{min}$. The retention time was found to be 7.8 minutes and $\lambda \max 330 \mathrm{~nm}$. For SCR7, the mobile phase consisted of a mix of acetonitrile and water $(1: 1)$ delivered at a rate of $1.0 \mathrm{~mL} / \mathrm{min}$. Retention time was 4.3 minutes and $\lambda \max 220$ $\mathrm{nm}$. 


$$
\text { Encapsulation Efficiency }(\mathrm{EE})=\frac{\text { Residual amount of drug in wafer }}{\text { Feeding amount of drug }} \times 100
$$

\section{In vitro drug release study.}

Drug release was carried out in artificial cerebrospinal fluid (CSF) $(\mathrm{NaCl} 148 \mathrm{mM}, \mathrm{KCl}$ $3 \mathrm{mM}, \quad \mathrm{MgCl}_{2} \quad 0.8 \mathrm{mM}, \mathrm{CaCl}_{2} 1.4 \mathrm{mM}$, $\mathrm{Na}_{2} \mathrm{HPO}_{4} 1.5 \mathrm{mM}, \mathrm{NaH}_{2} \mathrm{PO}_{4} 0.2 \mathrm{mM}$, BSA 0.1 $\mathrm{mg} / \mathrm{mL}$ ) with $\mathrm{pH}$ maintained in the physiological range (7.1-7.3). Drug-loaded wafers were submerged in $5 \mathrm{~mL}$ of simulated CSF taken in plastic vials and kept in a shaking incubator at $37^{\circ} \mathrm{C}$ with stirring of $50 \mathrm{rpm}$. Next, $0.5 \mathrm{~mL}$ of the release media was collected at specific time intervals and replenished with an equal volume of fresh media. HPLC was performed for each sample and the cumulative drug concentration was estimated from the standard curve equation.

\section{Statistics.}

Graphs were generated and statistical analysis was done using OriginPro 8 software (Version 8.0724, Origin Lab Corporation, Northampton, USA). $\mathrm{P}<0.05$ was considered as statistically significant.

\section{Results and discussions \\ SCR7 enhanced TMZ induced cytotoxicity and apoptosis}

Preliminary assessment of TMZ+SCR7 combination therapy was studied using MTT assay. First, we tested the cytotoxicity of free TMZ, SCR7 and TMZ+SCR7 combination on two different glioma cell lines, rat C6 and human MGMT over-expressing T98G. Untreated cells served as control. For TMZ, IC 50 was $550 \mu \mathrm{M}$ for C6 cells (Figure 1A) and $\sim 150 \mu \mathrm{M}$ for SCR7 (Figure 1B). However, when $550 \mu \mathrm{M}$ TMZ was used in combination with $100 \mu \mathrm{M}$ of SCR7 (IC90 ), the response was almost $80 \%$ cell toxicity for C6 cells. Similar effect was observed for MGMT over-expressing T98G cells too. Further, we confirmed the cell toxicity using apoptosis assay (Annexin V-PI) in flow cytometry. Untreated cells served as negative control and camptothecin-treated cells as the positive control. After 72 hours of drug treatment, $76.7 \%$ cells were live in the SCR7 treatment group. On the other hand, TMZ induced a better cytotoxicity and only $22.4 \%$ were alive. Combination therapy (TMZ+SCR7) significantly improved the toxicity, leaving only $7.2 \%$ cells alive (Figure 2D), after the treatment.
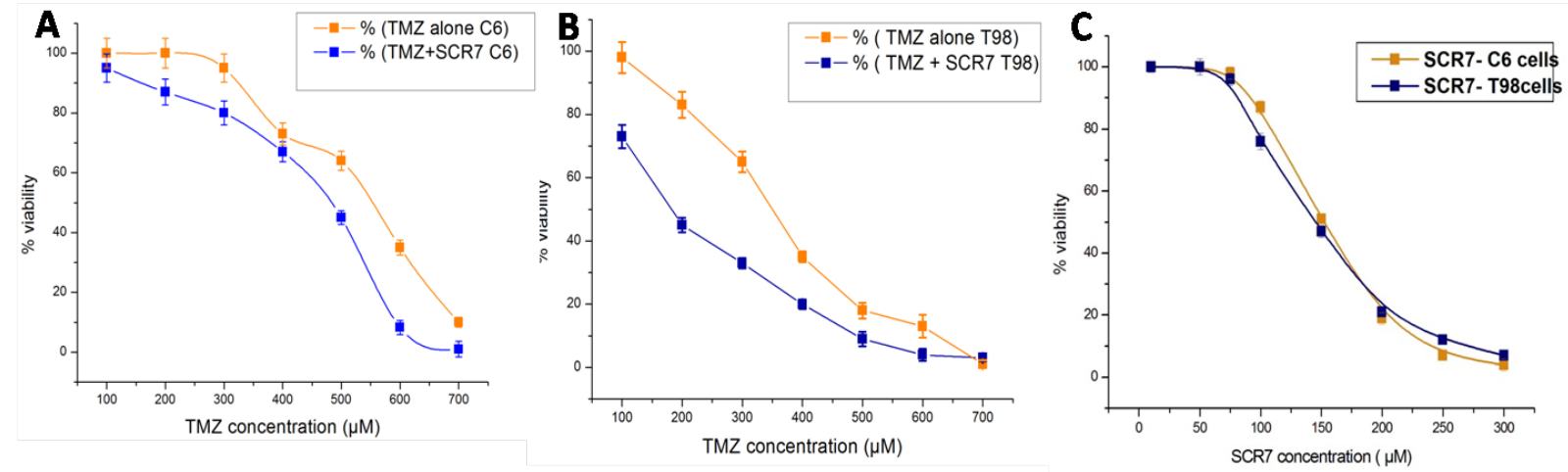

Figure 1. TMZ and TMZ+SCR7 dose response curve on C6 cells (A) and T98 cells (B) shows enhanced toxicity with $T M Z+S C R 7$ combination (C) $S C R 7$ dose response curve. ${ }^{*} P<0.05$ 
Combinatorial effect of free drugs on neurospheres

Compared to typical glioma neurosphere assays, our aim was to create an in vitro tumor recurrent assay using 3D culture model. For this, first, we treated C6 cell lines with chemotherapy drugs of interest, TMZ $(550 \mathrm{uM})$, SCR-7 $(100 \mathrm{uM})$ or their combination and cells remaining in the live quadrant were further cultured for their ability to grow back and forms colonies. These residual cells were cultured in serum free DMEM F12 media, supplemented with neural growth factors for a period of 12 days. As seen in Figure 2E, in untreated control, aggressive growth of colonies with cell to cell contact was seen within 3-12 days of culture. The spheroids were stained with LIVE/DEAD (CalceinAM/EthD-1) assay to study the viability of cells within the colony. Calcein is a nonflourescent dye, but after binding to cells gets converted into fluorescent calcein AM whereas EthD-1 binds to necrotic cell-DNA giving red fluorescence. Cells in the neurospheres were stained with calcein indicating live cells throughout the colony (Figure $2 \mathrm{~A} \mathrm{i}$ and ii). H\&E staining shows spheroid cells with filopodial extensions and stained uniformly throughout (Figure 2A iii). We used nestin staining to examine the stemness of the cells. Nestin is a marker for identifying neural progenitor cells. ${ }^{36}$ Neurospheres were stained positive for nestin indicating the presence of neural precursor cells (Figure 2B).

Effect of TMZ (550uM) alone in spheroid recurrence is shown in Figure 2E. The cells cultured from the live quadrant could easily repopulate and form active spheroids by days 9-12. Many active small colonies where seen around the large colony indicating a clear case of recurrence. In SCR 7 treated group, by day 3 itself, the spheres started forming. By days 6-12, large interconnected tumor spheres could be seen. This indicates that Ligase IV inhibition alone has no specific impact on the tumor cells. However, when SCR7 was combined with $\mathrm{TMZ}$, the colony formation was found again delayed to days 9 and 12 . The study was terminated on day 12 as both the control and SCR7 treated spheroids were over confluent and media depleted. MTT assay performed at each time point quantitatively measured the cell growth (Figure 2C) and showed that, compared to the untreated control, SCR7 and TMZ group, the rate of cell proliferation was relatively restricted in TMZ-SCR7 combination. However, from the TMZ-SCR7 cultures it is evident that although the metabolic activity was reduced, the free drug combination could not effectively inhibit spheroid re-growth which is the prime reason for tumor recurrence in vivo. We believe that, the free drug combination could not provide sustained cytotoxic stress to the spheroid cells and some of the refractory cells could recover over a period of few days. Next, we tested whether prolonged and sustained release of both the drugs using a slow-releasing polymer implant can overcome this challenge.

Preparation and characterization of polymeric wafer for combinatorial sustained drug release

A biodegradable polymeric (PLGA-PLA) wafer loaded with TMZ, SCR7 and TMZ-SCR7 combination was prepared using a lyophilization method (Figure 3A). Different PLGA: PLA concentrations were tried and the combination with desired release kinetics and physical properties such as flexibility, porosity, strength etc., was selected for the experiments (25:75). SEM analysis revealed the formation of highly uniform porous interconnected morphology of the wafers. The pore sizes ranged from 10 to $50 \mu \mathrm{m}$ (Figure 3B). Surface porosity play a key role in drug release and polymer degradation, especially in the case of bulk eroding polymers like PLGA. The presence of porous structure can help in enhanced penetration of physiological fluids leading to better drug release and polymer degradation by hydrolysis. ${ }^{37}$ The elemental mapping of TMZ and SCR7 wafers (Figure 3C $\& D$ respectively) showed uniform distribution of drugs throughout the wafer. Energy Dispersive Spectral (EDS) analysis enabled the qualitative mapping of the element over the substrate. The nitrogen atoms present in the TMZ molecules were mapped on the TMZ-loaded wafer as shown in Figure 3E. Similarly, the nitrogen and sulphur atoms present in the SCR7 were mapped on SCR-7 loaded wafer as shown in Figure 3F. Uniform distribution of the drugs in carrier matrix was important for obtaining reproducible controlled release. FTIR spectra of free drug as well as drug-loaded wafers, depicted in Figure $3 \mathrm{G}$, showed the characteristic vibration of TMZ molecule at $1610 \mathrm{~cm}^{-1}$ due to $\mathrm{C}=\mathrm{C}$ or $\mathrm{C}=\mathrm{N}$ stretching vibration. The $\mathrm{N}-\mathrm{H}$ 
stretching vibration at $3400 \mathrm{~cm}^{-1}$ was not so evident in the TMZ wafer. FTIR spectra of SCR7 and SCR7 wafer showed C-S stretching vibrations at $3271 \mathrm{~cm}^{-1}, \mathrm{C}-\mathrm{S}$ bending at $1082 \mathrm{~cm}^{-1}$ whereas N-H bending vibrations at $924 \mathrm{~cm}^{-1}$.
A

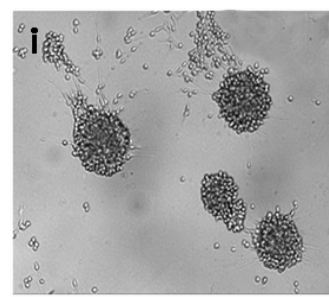

B

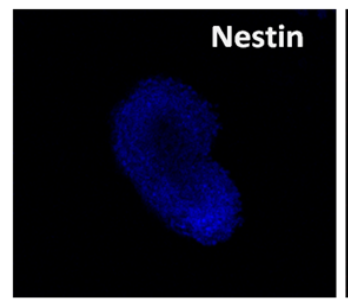

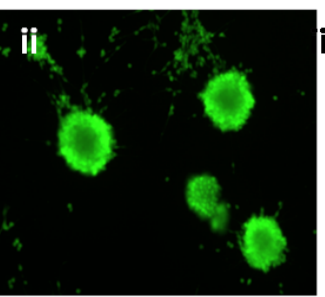

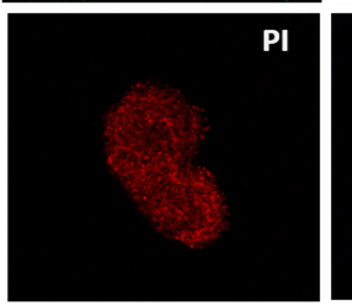

D

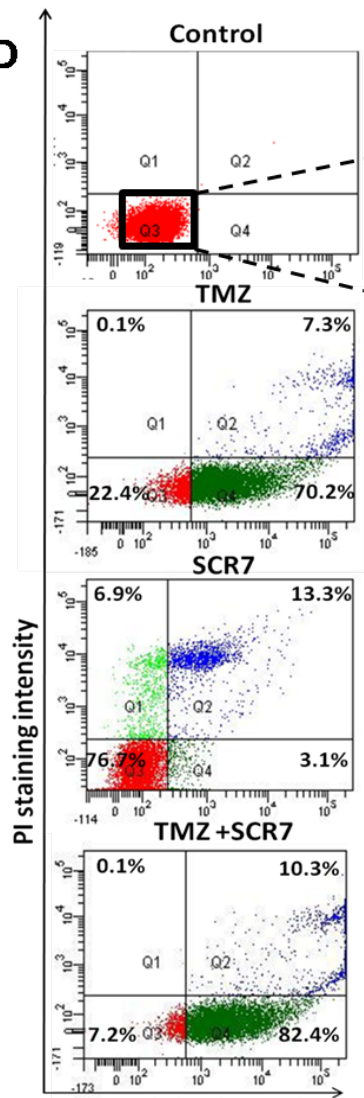

$\mathbf{E}$

Day 3

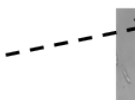

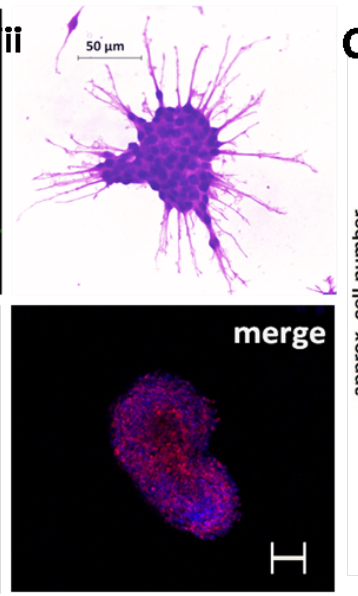

Day 6
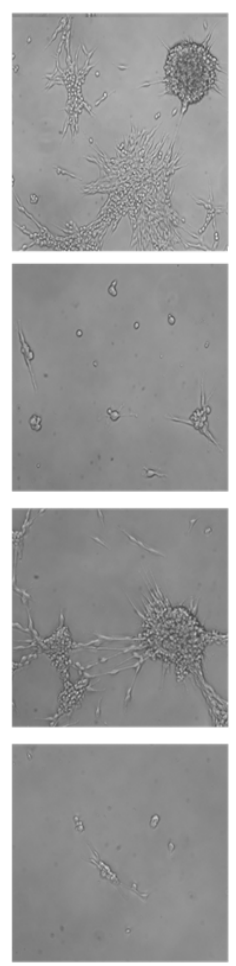

C

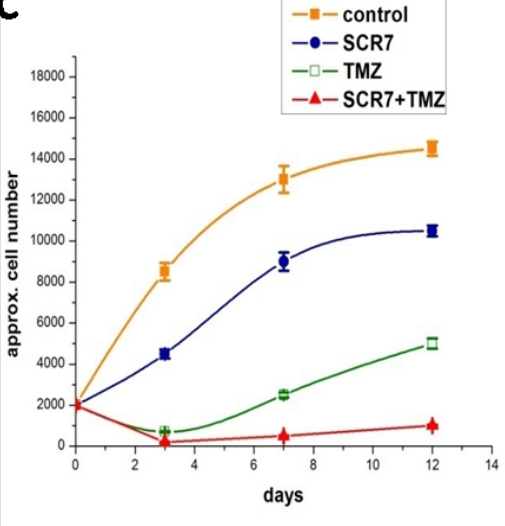

Day 9
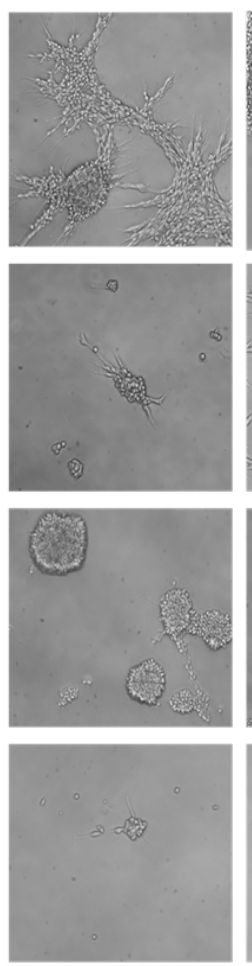

Day 12
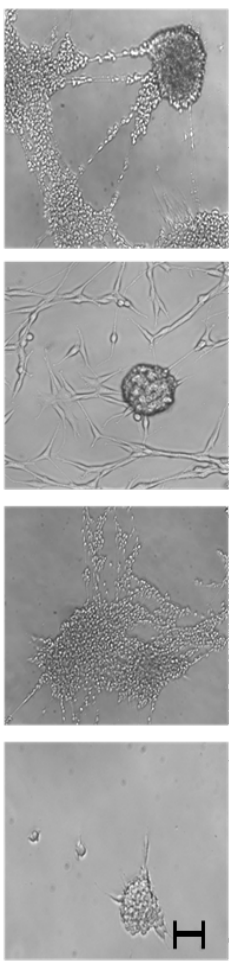

FITC staining intensity

Figure 2 (A) (i \& ii) Bright field and fluorescent images of Live/Dead staining showing live cells stained with calcein throughout the spheroid. (iii) H\&E staining of spheroid showing filopodial extensions and uniform staining (B) Nestin staining shows colonies stained positive for nestin indicating presence of neural progenitor cells [both A \& B -Scale bar $100 \mu \mathrm{m}$ ]. (C) Cell growth curve from MTT assay at various time points of cells treated with free drugs SCR-7 alone, TMZ alone, or $T M Z+S C R 7$ combination (D) flow cytogram showing enhanced apoptosis-mediated cell death by TMZ+SCR7 combination $(T M Z-550 \mu M, S C R 7-100 \mu M)(E)$ time dependent re-colonization of C6 cells in control, free drugs TMZ alone, SCR7 alone and TMZ+SCR7 treated groups showing resistant population proliferating and forming colonies [Scale bar 50 $\mu \mathrm{m}]$. 
A

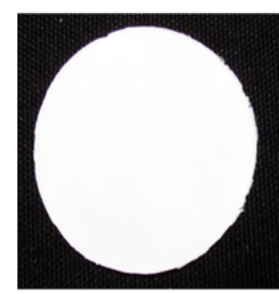

TMZ Wafer

C
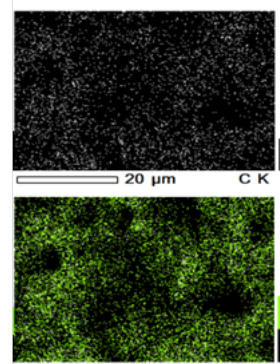

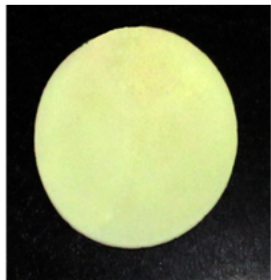

SCR7 Wafer
B

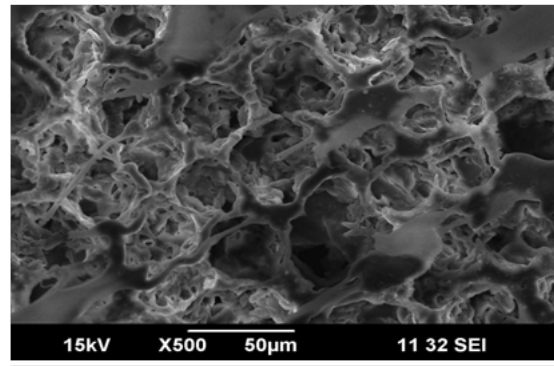

D
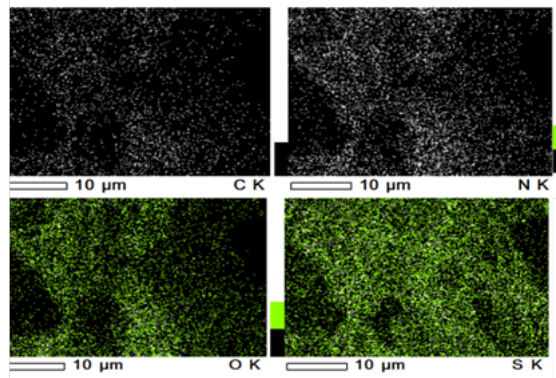

E
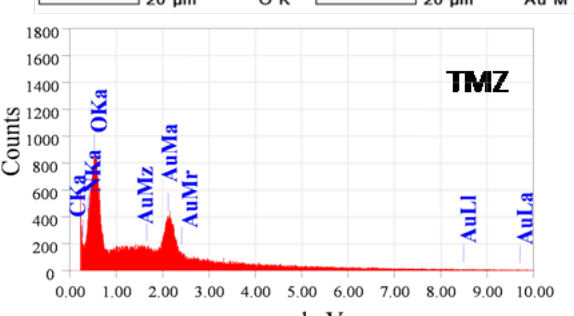
$\mathrm{keV}$

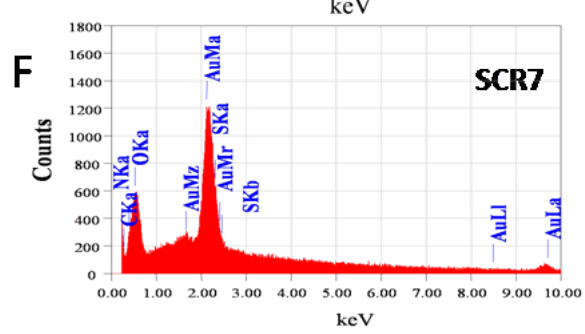

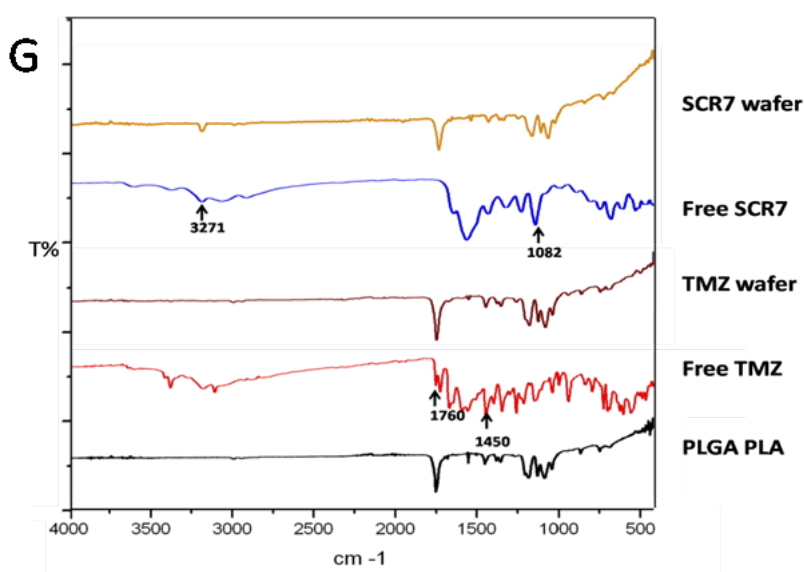

Figure 3. (A) Photographs of TMZ and SCR7 loaded wafers. (B) SEM image of wafer shows uniform and interconnected pores. $(\boldsymbol{C} \& \boldsymbol{E})$ elemental mapping and EDS spectrum of TMZ-loaded wafer respectively shows the uniform distribution of drugs throughout the wafer. (D \& $\boldsymbol{F})$ elemental mapping and EDS spectrum of SCR7 loaded wafer respectively showing uniform distribution of SCR7 throughout the wafer. (G) FTIR spectrum of bare wafer, free drugs and drug-loaded wafers.

Encapsulation Efficiency and in vitro drug release profile

HPLC analysis was carried to determine the encapsulation efficiency of drugs in the wafers. The supernatant obtained upon dissolving a known amount of wafer by phase separation process was analyzed using HPLC. While studying the release profile, the poor stability of TMZ in culture media was taken into account. A cumulative drug concentration is plotted for each time point after calculating the concentration of TMZ and its degradation product AIC (5-aminoimidazole-4-carboxamide), which is a stable compound, using HPLC. Nearly 70 and $72 \%$ encapsulation efficiency was obtained for both TMZ and SCR7, respectively. The drug release behavior of the wafer was carried in artificial CSF for a period of one month. The TMZ wafer showed almost $40 \%$ release in the first day itself indicating burst release. However, the release rate was controlled by optimizing the polymer composition and thus extended release was obtained up to one month (Figure 4A). The initial burst release of TMZ was not modified deliberately because our plan was to check the ability of tumor cells to recur even after the TMZ treatment. In the clinical scenario too, moderate burst release of drug may benefit the therapy by providing a relatively high dosage to the residual tumor cells in the tumor resected 
margin. In SCR7 loaded wafer, initial release was $10 \%$ followed by sustained release over a period of one month. The slow release was attributed to high hydrophobicity of SCR7 molecule, which might have facilitated strong hydrophobic interaction between SCR7 and PLGAPLA polymer blend.

\section{In vitro toxicity assessment of drug-loaded} wafers

In vitro cytotoxicity of drug-loaded wafer was studied in comparison with bare wafer or free drug combination. Figure 4B shows cell viability (alamar blue) assay where TMZ, SCR7 and their combinations were tested. Clearly, the drug combination showed significant enhancement in the cytotoxicity compared to individual drugs. In addition, the wafer loaded drugs showed better toxicity than free drugs. TMZ, which is a pro-drug, at physiological $\mathrm{pH}$ will spontaneously hydrolyze to a relatively unstable compound 5-(3-methyltriazen-1-yl) imidazole-4-carboxamide (MTIC), then to 5-aminoimidazole-4-carboxamide (AIC) and a highly reactive methyldiazonium ion that cause DNA methylation. ${ }^{38}$ This ion will be active only for a short period of time, restricting the half-life of TMZ in plasma to 1.8 hours. During systemic delivery, the concentration of TMZ in plasma will decrease exponentially, making repeated, daily dosing a necessity. The loading of TMZ into a locally implantable polymeric wafer will prevents its hydrolysis until it is released in brain, effectively increasing its half-life in the tumor micro-environment.
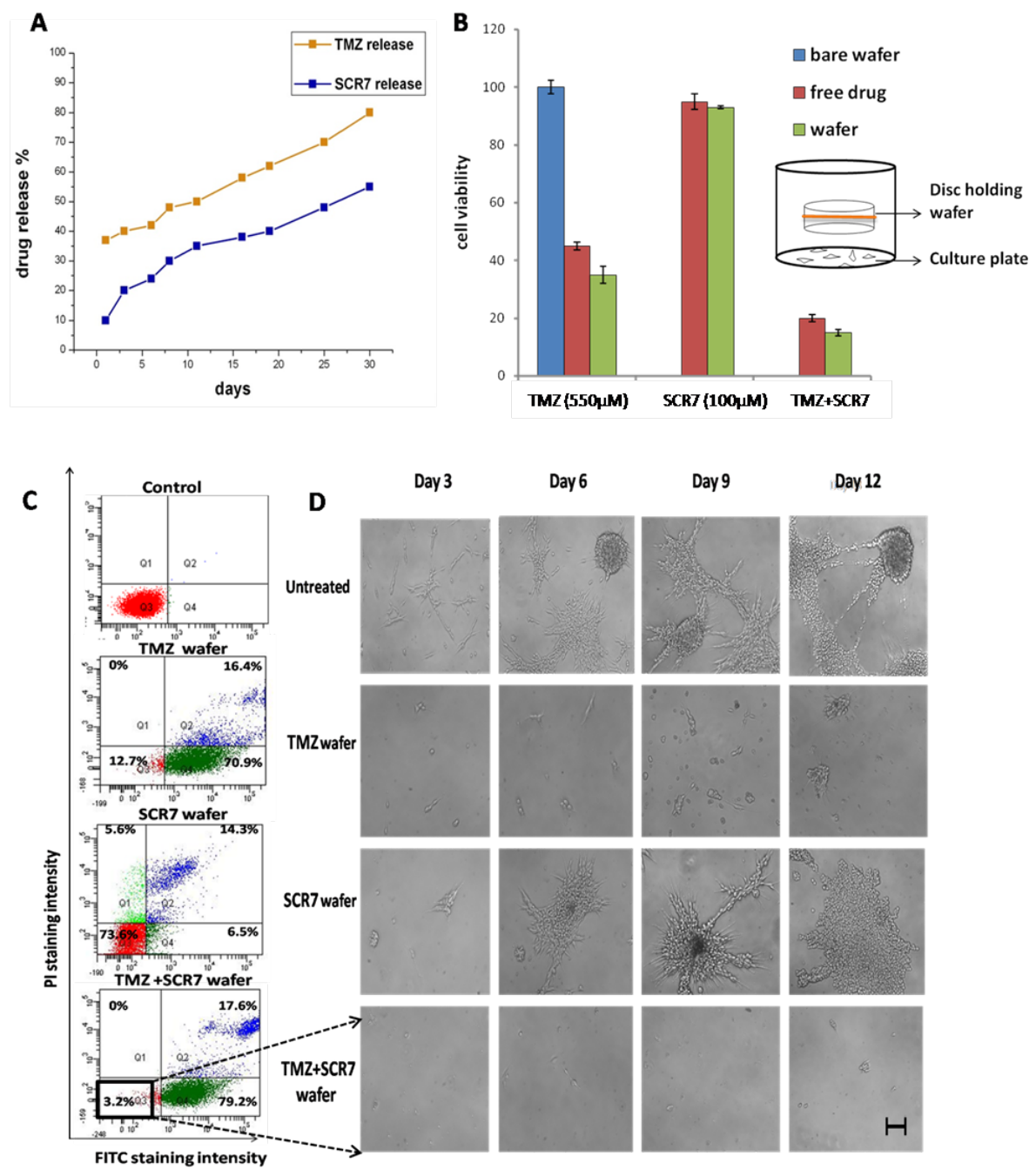

Figure 4. (A) In vitro drug release profile of TMZ and SCR7 loaded wafers in simulated CSF (B) In vitro cytotoxicity of drug-loaded wafer in comparison with bare wafers and individual drugs. Diagrammatic representation of the disc holding the wafer. The disc will hold wafer immersed in the media $(\boldsymbol{C})$ Flow cytogram showing apoptosis-mediated enhanced cell death by wafers. (D) Time dependent re-colonization of C6 cells in control, TMZ wafer, SCR7 wafer and TMZ+SCR7 wafer treated groups [Scale bar- $50 \mu \mathrm{m}$ ]. 
And thus, the cancer cells will be in contact with the active form of drug for relatively longer durations thereby enhancing the cytotoxicity. This was further validated by measuring apoptosis using flow cytometry (Figure 4C). In the TMZ-loaded wafer, $12.7 \%$ cells remain live compared to $22.4 \%$ in the free-TMZ control. The SCR7 wafer showed $73.8 \%$ live cells, almost same as that of free drug treated control (76.7\%). However, the TMZ+SCR7 combination wafer showed only $3.2 \%$ live cells which was $7.2 \%$ in case of free drug combination (Figure 2D). Clearly, sustained delivery of combination of drugs had significant impact within 72 hours. This was further tested in neurosphere culture (Figure 4D).

In colony formation assay, the cells that remained live after the wafer treatment were used to test its capability to re-form colonies. As shown, in case of TMZ wafer, $12.7 \%$ of cells remained live and they could regain the capacity to form small colonies by day 6 and welldefined colonies could be seen by day 12 . In the case of the SCR7 wafer, nearly $73 \%$ of cells remain live and they could establish large colonies by days 6-12. Multiple colonies were found merged together to form millimetre scale tumor regions. However, in case of TMZ-SCR7 combination wafer, no colonies were found till day 12 and only few minute clusters of cells were seen on day 12 . In comparison, free drug combination had well-formed colonies by day 12 (Figure 2D). Clearly, the results show that sustained release of combination of drugs has major impact in controlling the ability of cells to re-grow and form microscopic tumor recurrence. More detailed dose optimization studies are warranted to obtain complete tumor recurrence. Most importantly, as evident from our results, it is clear that, even $3 \%$ of cells that remain live after the drug treatment can regain and slowly re-establish microscopic tumor colonies. This is a real-life scenario that we see in the clinical cases of glioma recurrence. Here, we established a simple in vitro neurosphere tumor recurrence assay to study glioma recurrence and test the efficacy of clinically relevant drugs like TMZ or novel drugs such as SCR7 and their combinations either in free form or implantable, sustained releasing systems. Our results clearly suggest that even if we use rational combination of drugs, it is critical to deliver them in a sustained fashion for prolonged periods for obtaining the real advantage of drug combinations and multi-drug-loaded polymeric implants has the potential to address this challenge.

\section{Conclusion}

In this study a brain implant-based combination therapy using DNA alkylating agent TMZ and ligase IV inhibitor SCR7 was used to address the issue of tumor recurrence in glioma. A synergistic effect was observed when TMZ was combined with emerging novel molecule, SCR7, which inhibited the mismatch repair protein ligase IV, thereby preventing the reversal of damages caused by TMZ, ultimately giving better toxicity. The cells that escaped lethal effect of drug were further cultured to study the re-initiation of neurosphere. In order to provide sustained release of both drugs, we developed a biodegradable microporous polymeric wafer by lyophilization technique. Over all the study showed that: (i) even 5-7\% cells that survive after TMZ treatment has the capacity to recur and form microscopic tumor colonies within 3-12 days of culture in vitro; (ii) TMZ or SCR7 alone as single agent drug or as sustained releasing wafer had insignificant impact on inhibiting the tumor recurrence; (iii) TMZ+SCR7 combination clearly showed enhanced effect in inhibiting the spheroid recurrence, when delivered as a sustained releasing wafer compared to free drug combination. Our study clearly demonstrates the potential of locally implantable, multi-drug-loaded polymer implants for addressing the challenge of tumor recurrence in glioma spheroid models.

\section{Acknowledgment}

Authors thank Dept. of Science and Technology (DST) for the financial support under the project "Clinical translation and new frontiers of medical nanosystems at Amrita Institute of Medical Sciences, Kochi” [SR/NM/TP-15/2016(G)]

\section{Conflict of Interests}

The authors declare no conflicts of interest. For signed statements, please contact the journal office: editor@precisionnanomedicine.com 
Quote this article as: Devassy G, Ramachandran R, Jeena K, Junnuthula VR, Gopinatha VK, Manju CA, Manohar M, Nair SV, Raghavan SC, and Koyakutty M, Simultaneous release of dual drugs from polymer nano-implant inhibits recurrence in glioblastoma spheroids, Precis. Nanomed. 2019;2(1):218229, https://doi/org/0.33218/prnano2(1).181122.1

\section{References}

1. Lipani, J. D.; Jackson, P. S.; Soltys, S. G.; Sato, K.; Adler, J. R.; Survival following cyberKnife radiosurgery and hypofractionated radiotherapy for newly diagnosed glioblastoma multiforme, Technol. Cancer Res. Treat. 2008, 7(3),249-55.

2. Lowenstein, P. R.; Castro, M. G.; Pushing limits of glioma resection using electrophysiologic brain mapping, J. Clin. Oncol. 2012, 30, 2437.

3. Cohen, A.; Holmen, S.; Colman, H.; IDH1 and IDH2 mutations in gliomas, Curr. Neurol. Neurosci. Rep. 2013, 13, 345 .

4. Labussier, M.; Sanson, M.; Idbai, A.; IDH1 gene mutations: a new paradigm in glioma prognosis and therapy, Oncologist. 2010, 15, 196-199.

5. Padfield, E.; Ellis, H. P.; Kurian, K. M.; Current therapeutic advances targeting EGFR and EGFRvIII in glioblastoma, Front Oncol. 2015, 5, 5.

6. Heimberger, A. B.; Suki, D.; Yang, D.; Shi, W.; Aldape, K.; The natural history of EGFR and EGFRvIII in glioblastoma patients, J Transl Med. 2005, 3, 38.

7. Qiu, Z.; Shen, D.; Chen, Y.; Yang, Q.; Guo, C.; Feng, B.; Chen, Z.; Enhanced MGMT expression contributes to temozolomide resistance in glioma stem like cells, Chin J Cancer. 2014, 33, 155.

8. Kitange, G. J.; Carlson, B. L.; Schroeder, M. A.; Grogan, P. T.; Lamont, J. D.; Decker, P. A.; Wu, W.; James, C. D.; Sarkaria, J. N.; Evaluation of MGMT promoter methylation status and correlation with temozolomide response in orthotopic glioblastoma xenograft model, Neuro-Oncology. 2009, 11, 281-291.

9. Steinmetz, A.; Schackert, G.; Malignant gliomas of brain and surgical limitations, Oncol. Res. Treat. 1996, 19,1 .

10. Ellis, H. P.; Greenslade, M.; Powell, B.; Spiteri, I.; Sottoriva, A.; Kurian, K. M.; Current challenges in glioblastoma, intra tumour heterogeneity, residual disease and models to predict disease recurrence, Front. Oncol. 2015, 5, 251.

11. Sengupta, A.; Cancelas, J. A.; Cancer stem cells; a stride towards cancer cure, J. Cell. Physiol. 2010, 225,7 .

12. Young, R. M.; Jamshidi, A.; Davis, G.; Sherman, J. H.; current trends in surgical management and treatment of adult glioblastoma, Ann. Transl. Med.2015, 3, 121.

13. Lathia, J. D.; Mack, S. C.; Hubert, E. E.; Valentim, C. L.; Rich, J. N.; cancer stem cells in glioblastoma, Genes Dev. 2015, 29, 1203-1217.

14. Eyüpoglu, I. Y.; Hore, N.; Savaskan, N. E.; Grummich, P.; Roessler, K.; Buchfelder, M.; Ganslandt, O.; Improving the extent of malignant glioma resection by dual intraoperative visualisation approach, PLoS One 2012, 7,e44885.

15. Zalutsk, M. R.; Targeted radiotherapy of brain tumours, Br. J. Cancer 2004, 90, 1469-1473.

16. Akbar, U.; Jones, T.; Winestone, J.; Michael, M.; Shukla, A.; Sun, Y.; Duntsch, C.; Delivery of temozolomide to the tumour bed via biodegradable gel matrices in a novel model of intracranial glioma with resection, J. Neuro-Oncol. 2009, 94,203-212.

17. Lawrence, Y. R.; Wang, M.; Dicker, A. P.; Andrews, D.; Curran, W. J.; Michalski, J. M.; Souhami, L.; Yung, W. K.; Mehta, M.; Early toxicity predicts long term survival in high grade glioma, Br. J. Cancer 2011, 104, 1365.

18. Komarov, N. L.; Boland, C. R.; Cancer: calculated treatment, Nature 2013, 499, 291.

19. Nonnenmacher, L.; Westhoff, M.; Fulda, S.; Massler, G. K.; Halatsch, M.; Engelke, J.; Simmet, T.; Corbacioglu, S.; Debatin, K.; RIST: a potent new combination therapy for glioblastoma, Int. J. Cancer 2015, 136, 173. 
20. Chandran, P.; Gupta, N.; Retnakumari, A. P.; Malarvizhi, G. L.; Keechilat, P.; Nair, S.; Koyakutty, M.; Simultaneous inhibition of aberrant cancer kinome using rationally designed polymer-protein core shell nanomedicine, Nanomedicine:NBM, 2013, 9, 1317-1327.

21. Narayanan, S.; Ullas, M.; Dehannathparambil, K. V.; Manzoor, K.; Bindhu, P.; Menon, D.; Sequential release of epigallocatechin gallate and paclitaxel from PLGA casein core shell nanoparticle sensitizes drug resistant breast cancer cells, Nanomedicine:NBM 2015, 11, 1399-1406.

22. Gopikrishna, J.; Prasanth, B.; Nair, S. V.; Menon, D.; Influence of surface passivation of methoxyestradiol loaded PLGA nanoparticles on cellular interactions, pharmacokinetics and tumour accumulation, Colloids Surf.,B 2017, 150, 242-249.

23. Kumari, A.; Yadav, S. K.; Yadav, S. C.; Biodegradable polymeric nanoparticles-based drug delivery systems, Colloids Surf., B 2010, 75, 1.

24. Mangraviti , A.; Gullotti, D.; Brem, H.; Nanobiotechnology based drug delivery strategies; new frontiers in brain tumour targeted therapies, J. Controlled Release 2016, 240, 443-453.

25. Chowdhary, S. A.; Ryken, T.; Newton, H. B.; Survival outcome and safety of carmustine wafers in treatment of high grade gliomas: a meta-analysis, J. Neuro-Oncol. 2015, 122, 367-38.

26. Zhang, J.; Stevens, M. F.; Bradshaw, T. D.; Temezolomide mechanism of action, repair and resistance, Curr. Mol. Pharmacol.2012, 1, 102.

27. Ramachandran, R.; Junnuthula, V. R.; Gowd, G. S.; Ashokan, A.; Thomas, J.; Peethambaran, R.; Thomas, A.; Unni, A. K. K.; Panickar, D.; Nair, S. V.; Koyakutty, M. K.; Theragnostic 3 dimensional nano brain implant for prolonged and localised treatment of recurrent glioma, Sci. Rep. 2017, 7,43271.

28. Agarwala, S.S.; Kirkwood, J. M.; Temezolomide, a novel alkylating agent with activity in the central nervous system may improve the treatment of advanced metastatic melanoma, Oncologist.2000, 5, 144.

29. Spence, A. M.; Peterson, R. A.; Scharnhorst, J. D.; Silbergeld, D. L.; Rostomily, R. C.; Phase II study of concurrant continuous Temezolomide and Tamoxifen for recurrent malignant astrocytic gliomas, J. Neurooncol 2004, 70, 91.

30. Villano, J. L.; SeeryLinda, E.; Bressler, R.; Temezolomide in malignant gliomas; current use and future targets, Cancer Chemother. Pharmacol. 2009, 64, 647.

31. Srivastava, M.; Nambiar, M.; Sharma, S.; Karki, S. S.; Goldsmith, G.; Hegde, M.; Kumar, S.; Pandey, M.; Singh, R. K.; Ray, P.; Natarajan, R.; Kelkar, M.; De, A.; Choudhary, B.; Raghavan, S. C.; An inhibitor of nonhomologous end joining abrogates double strand break repair and impedes cancer progression, Cell $2012,151,1474$.

32. John, F.; George, J.; Vartak, S.V.; Srivastava, M.; Hassan, P. A.; Aswal, V. K.; Karki, S. S.; Raghavan, S. C.; Enhanced efficacy of pluronic copolymer micelle encapsulated SCR7 against cancer cell proliferation, J. Biol. Chem. 2015, 15, 521-534.

33. Vartak, S.V.; Raghavan, S. C.; Inhibition of nonhomologous end joining to increase the specificity of CRISPR/Cas9 genome editing, FEBS. J. 2015, 13416.

34. Tomkinson, A. E.; Howes, T. R.L.; Wiest, N. E.; DNA ligases as therapeutic targets, Transl. Cancer Res. 2013, 2, 1219.

35. Maruyama, T.; Dougan, S. K.; Truttmann, M. C.; Bilate, A. M.; Ingram, J. R.; Ploegh, H. L.; Increasing the efficiency of precise genome editing with CRISPR/Cas9 by inhibition of no homologous end joining, Nat. Biotechnol. 2015, 33, 538.

36. Chen, H.L; Yuh, C.H.; Wu, K. K.; Nestin is essential for zebra fish brain and eye development through control of progenitor cell apoptosis, PLoS One 2010, 5, e9318.

37. Lao, L.L.; Peppas, N. A.; Boey, F. C.; Venkatraman, S. S.; Modeling of drug release from bulk degrading polymers, Int. J. Pharmaceutics. 2011, 418, 28-41.

38. Baker, S. D.; Statkevich, W. M.; Reidenberg, P.; Alton, K.; Sartorius, S. E.; Dugan, M.; Cutler, D.; Batra, V.; Grochow, L. B.; Donehower, R. C.; Rowinsky, E. K.; Absorption, metabolism and excretion of 14ctemezolomide following oral administration to patients with advanced cancer, Clin. Canc. Res. 1991, 5, 309. 